\title{
Adaptive surrogate model based multi-objective transfer trajectory optimization between different libration points
}

\author{
Haijun Peng ${ }^{* a}$ and Wei Wang ${ }^{\mathrm{b}}$ \\ ${ }^{\mathrm{a}}$ Department of Engineering Mechanics, State Key Laboratory of Structural Analysis for Industrial \\ Equipment, Dalian University of Technology, Dalian, China \\ ${ }^{\mathrm{b}}$ Department of Engineering Mechanics, Tsinghua university, Beijing, China
}

\begin{abstract}
An adaptive surrogate model-based multi-objective optimization strategy that combines the benefits of invariant manifolds and low-thrust control toward developing a low-computational-cost transfer trajectory between libration orbits around the $L_{1}$ and $L_{2}$ libration points in the Sun-Earth system has been proposed in this paper. A new structure for a multi-objective transfer trajectory optimization model that divides the transfer trajectory into several segments and gives the dominations for invariant manifolds and low-thrust control in different segments has been established. To reduce the computational cost of multi-objective transfer trajectory optimization, a mixed sampling strategy-based adaptive surrogate model has been proposed. Numerical simulations show that the results obtained from the adaptive surrogate-based multi-objective optimization are in agreement with the results obtained using direct multi-objective optimization methods, and the computational workload of the adaptive surrogatebased multi-objective optimization is only approximately $10 \%$ of that of direct multi-objective optimization. Furthermore, the generating efficiency of the Pareto points of the adaptive surrogate-based multi-objective optimization is approximately 8 times that of the direct multi-objective optimization. Therefore, the proposed adaptive surrogate-based multi-objective optimization provides obvious advantages over direct multi-objective optimization methods.
\end{abstract}

Keyword: Surrogate model; Adaptive sampling; Multi-objective optimization; Libration point; Optimal control; Invariant manifold

Haijun Peng* (Corresponding author), E-mail: hjpeng@dlut.edu.cn

1

(C) 2016. This manuscript version is made available under the Elsevier user license http://www.elsevier.com/open-access/userlicense/1.0/ 


\section{Introduction}

With the increasing exploration of deep space, the significant value of libration points has been recognized by an increasing number of researchers (Cai et al., 2014; Peng et al., 2011a; Vaquero and Howell, 2014; Zhao and Cai, 2008). Libration points are not only ideal positions for interferometer and space telescopes but also excellent midway stations for long-term journeys. As interest in libration point orbits continues to increase and more missions utilize these orbits, a fuel-efficient method for constructing transfers between unstable libration point orbits will come to represent a powerful mission design tool. The ability to transfer spacecraft between libration point orbits will increase the number of options and flexibility in mission design (Davis et al., 2010; Davis et al., 2011).

Naturally, invariant manifolds that are associated with periodic orbits around libration points provide a good means for designing transfer trajectories between different libration point orbits. The invariant-manifold approach, which is based on dynamical system theory, has been regarded theoretically as a zero-cost transfer for the ideal case (Gómez et al., 1993). For example, Genesis is the first mission to be fully designed using dynamical system theory (Serban et al., 2002). However, periodic orbits around libration points in general may have no natural invariant manifolds intersecting with each other, and the time costs suffered by spacecraft along invariant manifolds are most likely unacceptable. On the other hand, low-thrust transfer technology, which is a direct transfer method, is of growing interest in spacecraft orbit mission design (Peng et al., 2014; Qi and Xu, 2015). Low-thrust propulsion enables higher velocity increments and greater payloads than traditional chemical propulsion. For example, the Deep Space (Rayman et al., 2000) and Dawn (Dankanich, 2010) missions have been executed using lowthrust propulsion. Therefore, low-thrust transfer can be employed and combined with invariant manifolds to overcome these drawbacks, and multi-objective (time cost, fuel cost, etc.) transfer trajectory design has become an important and valuable trend in recent years.

Many studies have expended substantial effort into addressing the multi-objective transfer trajectory design problem. Coverstone-Carroll et al. (2000) use a hybrid optimization method that combines a multi-objective genetic algorithm and a calculus-of-variation-based low-thrust trajectory optimizer to obtain the optimal trajectories of the Earth-Mars and Earth-Mercury missions. Schütze et al. (2009) employ a space pruning technique and subdivision technology to obtain the multi-objective low-thrust gravity-assist trajectories for interplanetary and orbital transfer. Dellnitz et al. (2009) propose a novel 
three-step approach for designing multi-objective transfer trajectories and apply the optimal trajectories for a formation flight task. Coffee et al. (2011) introduce a multi-objective optimization approach for designing transfer trajectories by extracting initial solutions from invariant manifolds and improving these solutions through variational optimal control. Assadian and Pourtakdoust (2010) utilize the multiobjective genetic algorithm to obtain the Pareto-optimal trajectories in the restricted four-body problem. In general, the global optimization problem is solved using evolutionary algorithms such as genetic algorithms and particle swarm optimization. In each generation of evolutionary algorithms, thousands of locally optimal control problems are solved. Therefore, large-scale computational and simulation work must be performed, and the problem of computational cost becomes considerable.

Motivated by the trade-off between computational costs and high-fidelity analysis capabilities, surrogate models have proven to be effective methods for complex optimization problems (Forrester and Keane, 2009; Gano et al., 2006; Pehlivanoglu and Yagiz, 2012; Peng et al., 2013). Because the surrogate model uses an explicit or approximate expression to replace the true complex model, optimization based on the surrogate model obtains a very high efficiency. As a result, the surrogate model has been shown to be an effective approach to the design of computationally expensive models such as many optimizations in aerospace and aerospace systems (Forrester and Keane, 2009; Pehlivanoglu and Yagiz, 2012). More recently, the surrogate model has been applied to the field of astrodynamics, including in Earth-Mars transfer orbit design, satellite constellations on low Earth orbit (Gano et al., 2006), and rendezvous on libration orbits (Peng et al., 2013).

To fully utilize the surrogate model, the methodology described in this paper follows a surrogate model-based multi-objective optimization strategy that combines the benefits of invariant manifolds and low thrust to develop a low-computational cost transfer trajectory between the Halo orbits around the $L_{1}$ and $L_{2}$ libration points in the Sun-Earth system. There are two principal differences from former studies, i.e., our new contributions. First, a new structure of the multi-objective transfer trajectory optimization model has been established. The methodology presented in most previous references wherein the transfer trajectories are designed by taking the invariant manifolds as the initial guesses of optimal control problems has not been used in this paper. The transfer trajectory now is broken down into several segments, and the invariant manifolds and the low thrust dominate different segments. Second, a mixed sampling strategy-based adaptive surrogate model has been proposed. Both the Pareto fitness function and the crowding distance function have been employed for constructing the mixed 
sampling strategy. Unlike the Pareto points directly obtained from the general surrogate model, they have not been tested using the real multi-objective optimization model. The present Pareto points obtained from the adaptive surrogate model have been verified through the real multi-objective optimization model.

This paper is organized as follows: In section 2, a brief review of the related background is introduced, including the dynamic model and the invariant manifolds associated with Halo orbits. In section 3, the multi-objective optimization problem is established for designing the transfer trajectory between the different libration points in the Sun-Earth system. In section 4, a mixed sampling strategybased adaptive surrogate model is proposed for solving the multi-objective optimization problem. In section 5, the performance of the adaptive surrogate model-based multi-objective optimization is verified through numerical simulations; in addition, some comparisons of multi-objective transfer trajectories and expanding applications are given in this section. Conclusions are drawn in section 6.

\section{Background}

\subsection{Dynamical model}

The circular restricted three-body model (CRTBP) is taken as a basic dynamical model in this paper for designing multi-objective transfer orbits. In the CRTBP, there are two massive bodies rotating around their barycenter with a constant angular velocity. Moreover, the spacecraft moves only under the effect of the two massive bodies, i.e., the Sun and the Earth, in this paper. A barycenter coordinate system is constructed whereby the original point is centered at the barycenter of the Sun-Earth system, the $x$-axis extends from the Sun to the Earth; the $y$-axis is in the rotational plane and normal to the $x$-axis; and the $z$-axis is defined using the rule of right-handed coordinate systems. The CRTBP model of the SunEarth system can be written in the following dimensionless form (Szebehely, 1967):

$$
\left\{\begin{array}{l}
\ddot{x}-2 \dot{y}=\frac{\partial U}{\partial x} \\
\ddot{y}+2 \dot{x}=\frac{\partial U}{\partial y} \\
\ddot{z}=\frac{\partial U}{\partial z}
\end{array}\right.
$$


where $U=\frac{1}{2}\left(x^{2}+y^{2}\right)+\frac{1-\mu}{r_{1}}+\frac{\mu}{r_{2}}+\frac{1}{2} \mu(1-\mu)$

The symbol $\mu$ is the ratio of the mass of the Earth over the sum of the masses of the Earth and Sun.

$r_{1}$ and $r_{2}$ are equal to the distances from the spacecraft to the Sun and to the Earth, respectively.

$$
\left\{\begin{array}{l}
r_{1}=\sqrt{(x+\mu)^{2}+y^{2}+z^{2}} \\
r_{2}=\sqrt{(x-1+\mu)^{2}+y^{2}+z^{2}}
\end{array}\right.
$$

\subsection{Halo orbits and invariant manifolds}

There are five well-known equilibrium solutions to the CRTBP, namely, the five Lagrange or libration points. Two linearly stable libration points compose equilateral triangles with the Sun and the Earth. Moreover, three unstable libration points lie on the $x$-axis of the barycenter coordinate system. Periodic and quasi-periodic orbits exist around unstable libration points. Halo orbits are 3-dimensional spatial periodic orbits around unstable libration points. It is well known that there are hyperbolic (stable and unstable) invariant manifolds associated with Halo orbits. Invariant manifolds contain stable manifolds $\left(W^{S}\right)$ and unstable manifolds $\left(W^{\mathrm{U}}\right)$. The orbits of the stable manifolds $W^{S}$ tend asymptotically to the Halo orbits, and the orbits of the unstable manifolds $W^{\mathrm{U}}$ diverge asymptotical

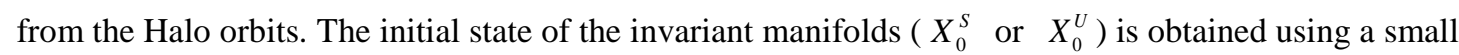
disturbance $d$ from the initial point $X_{0}$ on the Halo orbit. The initial state can be computed using the eigenvector of the monodromy matrix, and its physical meaning is the stable or unstable direction of the invariant manifold (Gómez et al., 2004), i.e.,

$$
X_{0}^{S}=X_{0} \pm d \frac{V^{S}}{\sqrt{V_{x}^{s}+V_{y}^{s}+V_{z}^{s}}} ; X_{0}^{U}=X_{0} \pm d \frac{V^{U}}{\sqrt{V_{x}^{U}+V_{y}^{U}+V_{z}^{U}}}
$$

where $V^{S}$ and $V^{U}$ are the stable and unstable eigenvectors, respectively, of the Halo orbit's state transition matrix. Moreover, in this paper, $d$ is chosen as $200 \mathrm{~km} / d_{\text {se }}$ (Gómez, et al, 1993) for computation of the invariant manifolds associated with Halo orbits around the $L_{1}$ and $L_{2}$ libration points in the Sun-Earth system (shown in Fig. 1). $d_{s e}$ is equal to $1.4959787 \times 10^{8} \mathrm{~km}$ and is the average distance between the Sun and the Earth. 

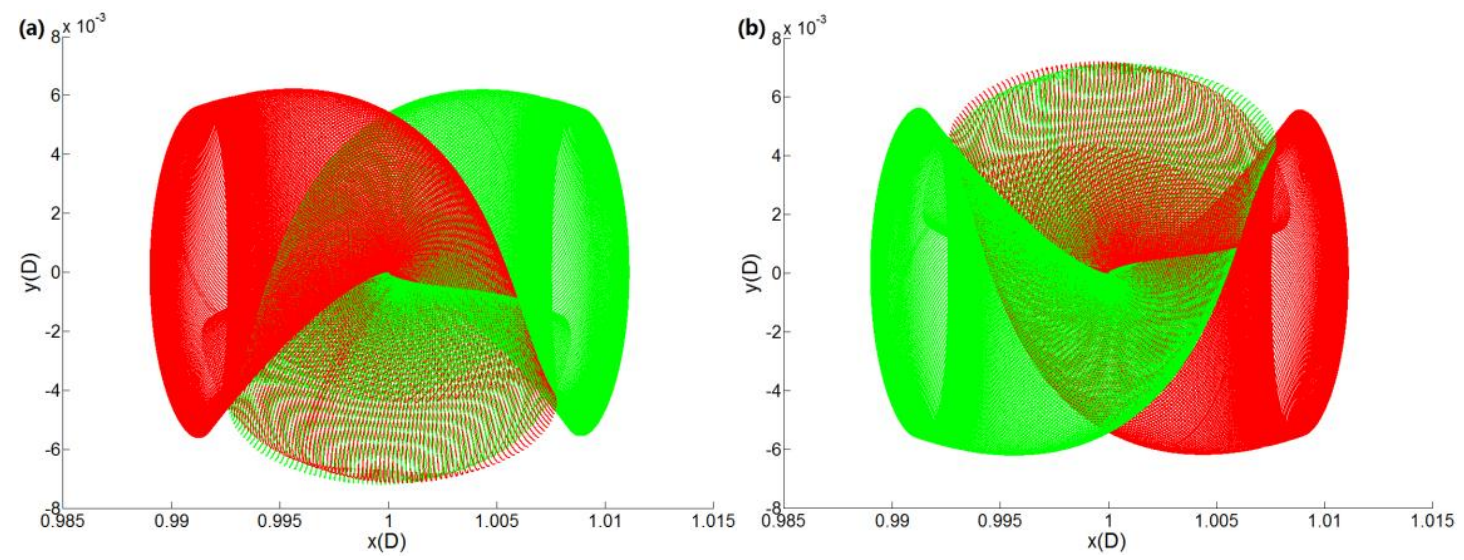

Fig.1. Invariant manifolds associated with Halo orbits around $L_{1}$ (left) and $L_{2}$ (right) libration points in the Sun-Earth system.

\section{Multi-objective transfer trajectory optimization problems}

\subsection{Optimal low-thrust transfer problem}

Many libration orbits do not have the same Jacobi constants; thus, there are no intersections for manifolds between these different libration orbits. The purpose of introducing optimal low-thrust transfer in this paper is to connect the disjoint invariant manifolds of Halo orbits around the $L_{1}$ and $L_{2}$ libration points in the Sun-Earth system.

We attempt to model the controlled motion of a spacecraft under the gravitational attractions of both the Sun and Earth as well as the low-thrust propulsion. The optimal low-thrust transfer problem can be constructed based on Eq. (1) and can be written in the state space as follows:

$$
\left\{\begin{array}{l}
\dot{x}_{1}=x_{4} \\
\dot{x}_{2}=x_{5} \\
\dot{x}_{3}=x_{6} \\
\dot{x}_{4}=2 x_{5}+x_{1}-(1-\mu) \frac{x_{1}+\mu}{r_{1}^{3}}-\mu \frac{x_{1}-1+\mu}{r_{2}^{3}}+u_{x} \\
\dot{x}_{5}=-2 x_{4}+x_{2}-(1-\mu) \frac{x_{2}}{r_{1}^{3}}-\mu \frac{x_{2}}{r_{2}^{3}}+u_{y} \\
\dot{x}_{6}=-(1-\mu) \frac{x_{3}}{r_{1}^{3}}-\mu \frac{x_{3}}{r_{2}^{3}}+u_{z}
\end{array}\right.
$$

where $u_{x}, u_{y}$ and $u_{z}$ are the control variables (acceleration in dimensionless form) in the $x, y$ and $z$ directions, respectively. The symbols $x_{1}=x, x_{2}=y, x_{3}=z, x_{4}=\dot{x}, x_{5}=\dot{y}$ and $x_{6}=\dot{z}$. 
The dot represents the time derivative in the rotating frame.

Eq. (4) can be rewritten in a compact explicit form as

$$
\dot{\mathbf{x}}=\mathbf{f}(\mathbf{x}(t), \mathbf{u}(t)) ; \mathbf{x}\left(t_{0}\right)=\mathbf{x}_{0}, \mathbf{x}\left(t_{f}\right)=\mathbf{x}_{f}
$$

where the symbol $\mathrm{f}$ denotes the vector field, $\mathbf{u}=\left(u_{x}, u_{y}, u_{z}\right)^{\mathrm{T}}$ is the control vector, $\mathbf{x}=\left(x_{1}, x_{2}, x_{3}, x_{4}, x_{5}, x_{6}\right)^{\mathrm{T}}$ is the state vector, $t_{0} / t_{f}$ are the initial/final times, $\boldsymbol{x}_{0}$ is the initial spacecraft state, and $\boldsymbol{x}_{f}$ is the final spacecraft state.

The transfer of a spacecraft from a Halo orbit around the $L_{1}\left(L_{2}\right)$ libration point to another Halo orbit around the $L_{2}\left(L_{1}\right)$ libration point is formulated as a nonlinear optimal control problem. The objective of the transfer mission is to minimize a performance measure of the following form:

$$
J=\int_{t_{0}}^{t_{f}} \Phi(\boldsymbol{x}(t), \boldsymbol{u}(t), t) d t
$$

where $\Phi(\boldsymbol{x}(t), \boldsymbol{u}(t), t)=\frac{1}{2} \mathbf{u}^{\mathrm{T}} \mathbf{u}$ is specified in this paper for energy-consumption minimization during the transfer process.

Thus, the optimal control problem for the design of the transfer trajectory can be summarized as follows: during a given fixed flight time $\left(t_{0}, t_{f}\right)$, a spacecraft under the dynamical model given by Eq. (5) is controlled from the initial conditions $\boldsymbol{x}_{0}$ to the final conditions $\boldsymbol{x}_{f}$ with the minimum energy consumption given by Eq. (6), and the above nonlinear optimal control problem is solved using symplectic algorithms (Peng et al., 2011b) in this paper to obtain an optimal low-thrust transfer trajectory.

\subsection{Multi-objective transfer trajectory optimization model}

With the continuous low-thrust and invariant manifolds in the Sun-Earth system, the multi-objective transfer trajectory optimization model is established in this section. For a spacecraft controlled using the mixed low-thrust and invariant-manifold approach that is transferred from a Halo orbit around the $L_{1}$ ( $L_{2}$ ) libration point to a Halo orbit around the $L_{2}\left(L_{1}\right)$ libration point in the Sun-Earth system, the transfer trajectory can be broken down into several segments (as shown in Fig. 2). In the first segment, the spacecraft starts from an unknown position on Halo orbits around the $L_{1}\left(L_{2}\right)$ libration point and travels on the unstable manifold. In the second segment, the spacecraft starts from the end of the first 
segment and is controlled via low-thrust propulsion to enter the stable manifold of the specified Halo orbit around the $L_{2}\left(L_{1}\right)$ libration point. Finally, the spacecraft travels on the stable manifold and arrives at the desired position.

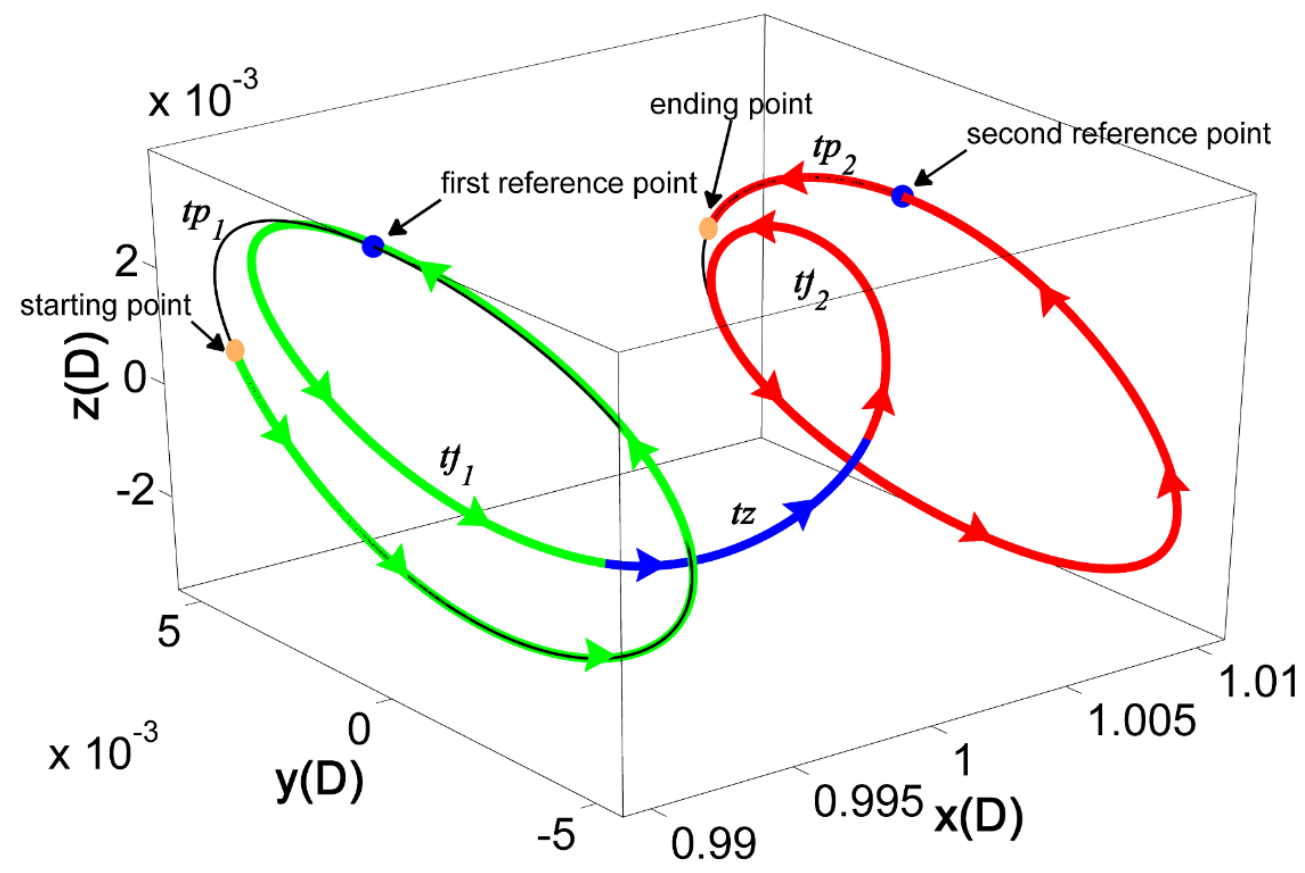

Fig.2. Geometry of transfer trajectories between Halo orbits around the $L_{1}$ and $L_{2}$ libration points

\subsubsection{Design variables}

Fig. 2 shows the basic geometry segments of mixed transfer trajectories between Halo orbits around the $L_{1}$ and $L_{2}$ libration points. Thus, there are 5 important parameters for determining the mixed transfer trajectories:

$t p_{1}$ : The flight time on the initial Halo orbit around the $L_{1}\left(L_{2}\right)$ libration point when it can determine the leaving state of the spacecraft. Because the initial condition of the Halo orbit has been given and taken as the first reference point, $t p_{1}$ can be used to determine the initial state $X_{0}$ of Eq. (3) for the diverging state of the unstable manifold.

$t f_{1}$ : The flight time on the unstable manifold of the initial Halo orbit around the $L_{1}\left(L_{2}\right)$ libration point. Because the parameter $d$ in Eq. (3) has been given, the flight time $t f_{1}$ required to diverge a Halo orbit from an orbit of its unstable manifold is finite. 
$t p_{2}$ : The flight time on the final Halo orbit around the $L_{2}\left(L_{1}\right)$ libration point when it can determine the arriving state of the spacecraft. Because the initial condition of the Halo orbit has been given and taken as the second reference point, $t p_{2}$ can be used to determine the initial state $X_{0}$ of Eq. (3) for the arriving state of the stable manifold.

$t f_{2}$ : The flight time on the stable manifold of the final Halo orbit around the $L_{2}\left(L_{1}\right)$ libration point. Because the parameter $d$ in Eq. (3) has been given, the flight time $t f_{2}$ required to arrive on a Halo orbit from an orbit of its stable manifold is finite.

$t z$ : The flight time for a spacecraft when it is controlled via low-thrust control and connects the unstable manifold of the initial Halo orbit and the stable manifold of the final Halo orbit.

The other parameters, including the initial and final states of the spacecraft in the second segment and the leaving/arriving states of the spacecraft on the specified Halo orbits, are associated with the optimization parameters. In addition, they can be computed based on the above parameters that have been selected for the multi-objective optimization.

\subsubsection{Constraints}

For a specific spacecraft transfer mission, all the above design variables should not take on arbitrary values and should satisfy practical constraint conditions.

First, this paper primarily focuses on the periodic Halo orbit around the $L_{1}$ and $L_{2}$ libration points in the Sun-Earth system, and the period $T_{\text {halo, initial }} / T_{\text {halo,final }}$ of the initial/final Halo libration point orbit is finite. Because different phases with an integer period may coincide with each other, the value of the flight time parameters $t p_{1}$ and $t p_{2}$ on the Halo libration point orbit at which the spacecraft leaves/arrives should be limited within one period. Therefore, the constraints on parameters $t p_{1}$ and $t p_{2}$ are

$$
0 \leq t p_{1} \leq T_{\text {halo,initial }}, 0 \leq t p_{2} \leq T_{\text {halo, final }}
$$

Second, either the unstable manifold of the leaving Halo orbit or the stable manifold of the arriving Halo orbit has different transfer times. In practical missions, the flight times $t f_{1}$ and $t f_{2}$ of a spacecraft moving on unstable/stable manifolds should not be very large, i.e., 


$$
0 \leq t f_{1} \leq t f_{1, \max }, 0 \leq t f_{2} \leq t f_{2, \max }
$$

Finally, the flight time $t z$ of the controlled spacecraft from the unstable manifold to the stable manifold is limited. The flight time $t z$ should satisfy the following constraints:

$$
t z_{\min } \leq t z \leq t z_{\max }
$$

It is noted that the basic and rough description of the constraints on the design variables have been given, and a detailed illustration and values of the above constraints for the specific multi-objective transfer missions have been listed in section 5 .

\subsubsection{Objective functions}

In the initial transfer trajectory design phases, many objectives should be considered. In the present mission, two objective functions, i.e., the total transfer time and the total fuel expenditure, are the two most important factors in the design of the transfer trajectory.

First, the total transfer flight time $\Delta t$ includes the flight times $t f_{1}$ and $t f_{2}$ of the spacecraft moving on unstable/stable manifolds and the flight time $t z$ of the spacecraft controlled using low-thrust control from the unstable manifold to the stable manifold, i.e.,

$$
\Delta t=t f_{1}+t z+t f_{2}
$$

Second, the fuel expenditure of the spacecraft is dependent on the controlled low-thrust segment. To measure the fuel expenditure for the controlled transfer time $t z$, the velocity increment $\Delta v$ is defined as follows (Peng et al., 2011a and 2013):

$$
\begin{gathered}
\Delta v=\sqrt{\Delta v_{x}^{2}+\Delta v_{y}^{2}+\Delta v_{z}^{2}} \\
\Delta v_{x}=\int_{t_{0}}^{t_{f}}\left|u_{x}\right| d t, \Delta v_{y}=\int_{t_{0}}^{t_{f}}\left|u_{y}\right| d t, \Delta v_{z}=\int_{t_{0}}^{t_{f}}\left|u_{z}\right| d t
\end{gathered}
$$

\subsubsection{Multi-objective optimization model}

From the above discussion, all the key factors in the optimization problem have been given. The multi-objective optimization model for transfer trajectories between Halo orbits around the $L_{1}$ and $L_{2}$ libration points in the Sun-Earth system can be summarized as follows: 


$$
\begin{cases}\text { find } & t p_{1}, t p_{2}, t f_{1}, t f_{2}, t z \\ \min & \Delta v, \Delta t \\ \text { s.t. } & t p_{1, \text { min }} \leq t p_{1} \leq t p_{1, \max } \\ & t p_{2, \text { min }} \leq t p_{2} \leq t p_{2, \max } \\ & t f_{1, \text { min }} \leq t f_{1} \leq t f_{1, \max } \\ t f_{2, \min } \leq t f_{2} \leq t f_{2, \max } \\ t z_{\min } \leq t z \leq t z_{\max } \\ \text { find } \boldsymbol{u}(t) \\ \min \quad J=\int_{t_{0}}^{t_{f}} \Phi(\boldsymbol{x}(t), \boldsymbol{u}(t), t) d t \\ \text { s.t. } \quad \dot{\boldsymbol{x}}=f(\boldsymbol{x}(t), \boldsymbol{u}(t), t) \\ \boldsymbol{x}\left(t_{0}\right)=\boldsymbol{x}_{0} \\ \boldsymbol{x}\left(t_{f}\right)=\boldsymbol{x}_{f}\end{cases}
$$

The multi-objective optimization model in Eq. (13) is parameterized by $t p_{1}, t p_{2}, t f_{1}, t f_{2}$ and $t z$. For each set of these quantities, the total velocity increment $\Delta v$ and the total time of flight $\Delta t$ necessary to send the spacecraft between the given periodic libration orbits are determined. In this mixed low-thrust and invariant-manifold transfer problem, a local single-objective optimal control problem must be solved in the inner layer of optimization; then, a global multi-objective optimization is implemented in the outer layer of optimization. Ultimately, the aim of the multi-objective optimization algorithm is to compute optimal values of these parameters to minimize both the total velocity increment $\Delta v$ and the total time of flight $\Delta t$. However, multi-objective optimization problems are usually solved using evolutionary algorithms, such as genetic algorithms; substantial computational work is necessary to obtain satisfactory design results. Furthermore, enormous nonlinear optimal control problems should also be evaluated in each sample of evolutionary algorithms. Therefore, it is necessary to propose an efficient optimization method for solving the above multi-objective optimization model.

\section{Adaptive surrogate model-based multi-objective optimization}

To overcome the difficulty presented by the computational burden, a multi-objective optimization method based on an adaptive surrogate model is proposed in this section to increase the computational efficiency for the present multi-objective transfer optimization problem. The key aspect of the adaptive surrogate model is to construct an approximate model to simulate the real response using some fitting technologies and apply an optimization method to the approximate model. This section first introduces the fitting method; then, the sampling strategy is proposed to update the surrogate model. The 
convergence criteria are used to evaluate the approximate model. The process of the adaptive surrogate model-based multi-objective optimization method is proposed in the last part.

\subsection{Construction of basic surrogate model}

The surrogate model, which is known as a mathematical model, is used to approximate the behavior of complex systems. This model is constructed using the input and output relationships of sample points. In general, the sample points are obtained from the design of experiments (DOE) (Queipo et al., 2005). If some DOE methods are applied in the input domain, the values of sample points are evaluated using the real response. A surrogate model can be constructed using these sample points. Many fitting technologies, such as polynomial regression, the Kriging method, and radial basis functions, can be used to construct the surrogate model. In this paper, the radial basis function method is employed to construct the basic surrogate model.

Radial basis functions use linear combinations of basis functions to express the objective function (Queipo et al., 2005). The basic expression formula can be expressed as follows:

$$
\hat{\mathbf{y}}(\mathbf{x})=\sum_{i=1}^{k} \beta_{i} \phi_{i}\left(\left\|\mathbf{x}-\mathbf{x}_{i}\right\|\right)=\boldsymbol{\beta}^{\mathrm{T}} \boldsymbol{\phi}
$$

where $\beta_{i}$ is the coefficient of the basis function $\phi_{i}, k$ is the number of evaluated sample points, and $\left\|\mathbf{x}-\mathbf{x}_{i}\right\|$ represents the Euclidean distance from $\mathbf{x}$ to the evaluated sample point $\mathbf{x}_{i}$. The basis function $\phi_{i}$ can be chosen as a Gaussian function, exponential function, multi-quadric function, etc. The exponential function is chosen in this paper.

With the radial basis function given in Eq. (14), the interpolation condition, namely, that the value of the approximation function should be equal to the real function at sample points, is satisfied as follows:

$$
\hat{\mathbf{y}}\left(\mathbf{x}_{i}\right)=\mathbf{y}\left(\mathbf{x}_{i}\right)
$$

where $\hat{\mathbf{y}}\left(\mathbf{x}_{i}\right)$ is the approximate value at the evaluated sample point $\mathbf{x}_{i}$ and $\mathbf{y}\left(\mathbf{x}_{i}\right)$ is the exact solution at the evaluated sample point $\mathbf{x}_{i}$.

With the interpolation condition Eq. (15), the radial basis function in Eq. (14) can be rewritten as follows:

$$
\mathbf{A} \boldsymbol{\beta}=\mathbf{y}
$$




$$
\begin{gathered}
\mathbf{A}=\left[\begin{array}{ccc}
\phi\left(\left\|\mathbf{x}_{1}-\mathbf{x}_{l}\right\|\right) & \cdots & \phi\left(\left\|\mathbf{x}_{1}-\mathbf{x}_{k}\right\|\right) \\
\vdots & \ddots & \vdots \\
\phi\left(\left\|\mathbf{x}_{k}-\mathbf{x}_{l}\right\|\right) & \ldots & \phi\left(\left\|\mathbf{x}_{k}-\mathbf{x}_{k}\right\|\right)
\end{array}\right] \\
\mathbf{y}=\left[\begin{array}{c}
\mathbf{y}\left(\mathbf{x}_{l}\right) \\
\vdots \\
\mathbf{y}\left(\mathbf{x}_{k}\right)
\end{array}\right]
\end{gathered}
$$

From the new expression of the radial basis function Eqs. (16) (18), the unknown coefficient matrix $\beta$ can be determined by solving the above linear equation, i.e.,

$$
\boldsymbol{\beta}=\mathbf{A}^{-1} \mathbf{y}
$$

Although the radial basis function is simply a linear combination of basis functions, the radial basis function method can also predict nonlinear responses of the optimization system by choosing nonlinear basis functions. Moreover, the distributions of sample points derived from DOE also have an important influence on the accuracy of the present surrogate model. Therefore, the mixed sampling strategy and adaptive implementation procedures are proposed in the following sections for constructing a higher accuracy surrogate model of multi-objective optimization.

\subsection{Mixed sampling strategy}

To improve the accuracy of the constructed surrogate model, more samples should be generated. To update the surrogate model, samples should be combined with the previous samples. This part attempts to find some samples that are close to the real Pareto region. In general, the multi-objective functions are conflicting, and optimization with respect to one objective function results in non-optimality with respect to the other functions. Therefore, we cannot find an optimal design that optimally satisfies all objective functions. In such cases, a Pareto front is the most optimal solution for the multi-objective optimization problem.

To choose the best sample points, a Pareto fitness function is defined as follows (Schaumann et al., 1998):

$$
F_{i}=1-\max _{j=1, \cdots, m, j \neq i}\left(\min \left(f_{p 1}^{i}-f_{p 1}^{j}, f_{p 2}^{i}-f_{p 2}^{j}, \cdots, f_{p n}^{i}-f_{p n}^{j}\right)\right)
$$

where $F_{i}$ denotes the value of the Pareto fitness function for the $i$ th $(i=1, \cdots, m)$ design point, $\quad f_{p q}^{i}$ is the value of the normalized Pareto fitness function for the $q$ th $(q=1, \cdots, m)$ objective function associated 
with the $i$ th design point, $n$ is the number of objective functions, and $m$ is the number of design points.

$$
f_{p 1}^{i}=\frac{r a w f_{p 1}^{i}-r a w f_{p 1}^{\min }}{r a w f_{p 1}^{\max }-r a w f_{p 1}^{\min }}
$$

where $\operatorname{rawf}_{p 1}^{i}$ is the value of the first objective function in the $i$ th design point, $\operatorname{rawf}_{p 1}^{\min }$ is the minimum value of the first objective function among all design points, and $r a w f_{p 1}^{\max }$ is the maximum value of the first objective function among all design points.

Obviously, every design point has a fitness value $F$, which can be employed to choose the best design points. Naturally, some basic facts can be obtained from the above definitions. First, the fitness values for non-Pareto-optimal points are in the range of 0-1, and these values for Pareto points are in the range of 1-2. Second, if the Pareto points are highly density and are evenly distributed, the value of the Pareto fitness function associated with these sample points should be approximately equal to 1 . Therefore, higher values of the Pareto fitness functions are computed from sample points, and improved Pareto points should be employed.

In this paper, the Latin hypercube design is taken as the initial DOE, and two sampling guidance functions are combined to form the presented mixed sampling strategy. The first sampling guidance function is the Pareto fitness function, and the purpose of the first sampling guidance function is to select the best approximate Pareto points. The second sampling guidance function is the crowding distance function (Deb et al., 2002), which can guarantee the uniformity of the approximate Pareto points.

For the first sampling guidance function, when the surrogate model is constructed, the nondominated sorting genetic algorithm (NSGA-II) (Deb et al., 2002) can be used to obtain the Pareto points of the surrogate model. These Pareto points obtained from the surrogate model are called pseudo Pareto points. These points are not the real Pareto points; however, they may be closer to the real Pareto frontier compared to other points. In general, a larger fitness value leads to a better sample point, and the sample points with large fitness values should be taken as the updated samples. Fig. 3 shows an illustrative example for the first sampling guidance function. Clearly, the middle interval, corresponding to the first part of the Pareto region, has a higher value of the Pareto fitness function than does the second part. 
(a)

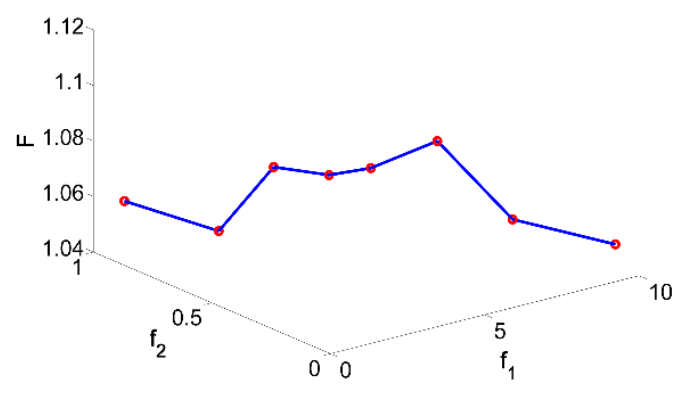

(b)

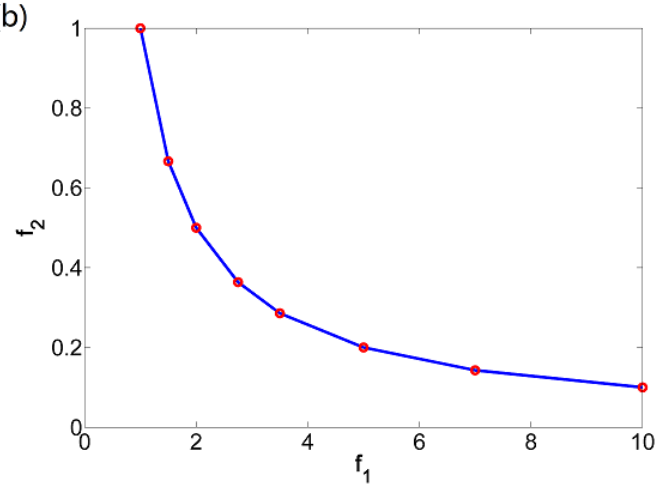

Fig. 3. An illustrative example of the first sampling guidance function; (a) the relationship between Pareto fitness functions and objective functions; (b) two objective function values

For the second sampling guidance function, to make the sample points evenly distributed, a concept called the crowding distance $c d$, which comes from NSGA-II, is proposed in this section:

$$
\begin{gathered}
c d_{i}=\min \left(\mathrm{d}_{i 1}, \mathrm{~d}_{i 2}, \cdots, \mathrm{d}_{i k}\right) \\
\mathrm{d}_{i j}=\sqrt{\left(\hat{f}_{p 1}^{i}-f_{a 1}^{j}\right)^{2}+\cdots+\left(\hat{f}_{p n}^{i}-f_{a n}^{j}\right)^{2}}
\end{gathered}
$$

where $\hat{f}_{p 1}^{i}$ is the normalized value of the first objective function, which is selected from the pseudo Pareto front. $f_{a 1}^{j}$ is also the normalized value of the first objective function; however, it is chosen from the present approximate Pareto points. $n$ is the number of objective functions, and $k$ is the number of current approximate Pareto points.

A larger crowding distance indicates that the selected sample point is not close to any approximate Pareto point, and this feature guarantees the uniformity of the sample points. When sample points with larger values of the crowding distance are selected, these sample points are taken into the multi-objective transfer optimization model to obtain the real responses. Moreover, the total number of updating sample points is related to the number of design variables, objective functions and current number of sample points. Therefore, the sample updating strategy is defined as follows:

$$
p_{\text {add }}= \begin{cases}5 & p_{a}^{i}-p_{a}^{i-1} \leq 0 \\ p_{a}^{i}-p_{a}^{i-1} & p_{a}^{i}-p_{a}^{i-1}>0\end{cases}
$$

where $p_{\text {add }}$ is the number of updated sample points and $p_{a}^{i}$ is the number of approximate Pareto points in the ith iteration.

Finally, two sampling guidance methods employ the same number of updated sample points for convenience. Thus, the total number of updated sample points is $2 \times p_{\text {add }}$ in each iteration. 


\subsection{Convergence criteria}

In the above mixed sampling strategy, two sampling guidance functions are employed for updating sample points. As a result, convergence criteria should be applied for finishing the mixed sampling strategy. Meanwhile, to fully utilize the updated sample points and provide sufficient choices for users to choose a suitable Pareto point, two convergence criteria are given in this work.

The first convergence criterion is the mean fitness value of the current approximate Pareto points; this value should be approximately equal to 1 .

$$
1 \leq \operatorname{mean}(\boldsymbol{F}) \leq F_{\max }
$$

where the parameter $F_{\max }$ is set to be slightly larger than 1 . Thus, the mean value of the Pareto fitness functions mean $(F)$ is approximately equal to 1 . Therefore, the current approximate Pareto points are close to each other and most likely exhibit good uniformity (Shan and Wang, 2005).

The second convergence criterion is the number of approximate Pareto points; this number is larger than a predesigned value.

$$
p_{a} \geq c
$$

where $p_{a}$ is the number of approximate Pareto points and $c$ is a given constant.

\subsection{Adaptive implementation procedures}

The main implementation procedures of the adaptive surrogate model based multi-objective transfer trajectory optimization can be summarized as follows:

Step 1: Initial DOE and construction of basic surrogate model. The Latin hypercube design is chosen to generate $n \times m^{2}$ sample points in the design space, where $n$ is the number of objective functions and $m$ is the number of design variables. These sample points are evaluated through the real multi-objective optimization model to obtain exact outputs. With these known initial sample points and the corresponding exact outputs, a basic surrogate model can be constructed using the radial basis function method.

Step 2: Obtain initial approximate Pareto points. The initial sample points are employed to evaluate the fitness function. Among these initial sample points, the values of the fitness function corresponding to certain sample points that are larger than 1 are chosen as the initial approximate Pareto points. 
Step 3: Obtain pseudo Pareto frontier. The multi-objective optimization problem formed by the explicit surrogate model is solved by the NSGA-II algorithm, and the Pareto frontier of the present surrogate model can be obtained.

Step 4: Update current approximate Pareto points. The mixed sampling strategy is utilized to generate the updated sample points. These sample points are evaluated using the real multi-objective transfer optimization model; then, they are combined with the current approximate Pareto points. In this step, all the combined points are evaluated using the multi-objective transfer model, the results can be used directly.

Step 5: Check convergence criteria. Two convergence criteria, i.e., the mean fitness value of the current approximate Pareto points and the number of current approximate Pareto points, are evaluated. If the convergence criteria have been satisfied, then we obtain the final multi-objective optimization results. Otherwise, the surrogate model should be updated for the next generation circulation.

Step 6: Update surrogate model. The sample points generated using the mixed sampling strategy in step 4 are combined with the present sample points to update the surrogate model. When a new surrogate model is constructed with new sample points, the process returns to step 3. 


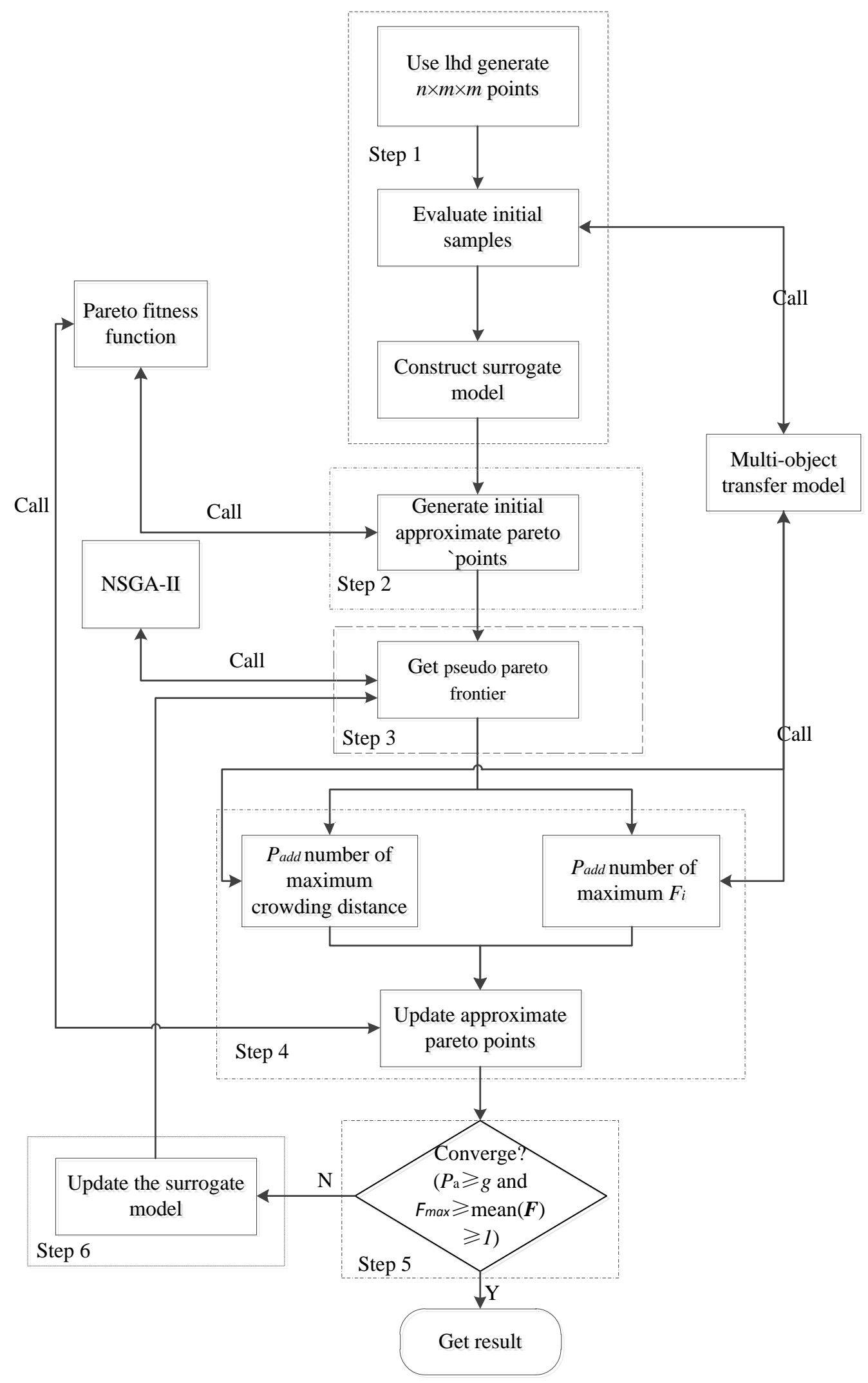

Fig. 4. Adaptive implementation flowchart of surrogate-based multi-objective optimization 


\section{Numerical simulations}

In this section, a spacecraft transfer design between Halo orbits of the $L_{1}$ libration point and Halo orbits of the $L_{2}$ libration point that employs invariant manifolds and low-thrust control in the Sun-Earth system is implemented in numerical simulations. The multi-objective transfer trajectories obtained using the adaptive surrogate model-based multi-objective optimization are provided as a proof of concept and as a basis for future investigations. All computations have been performed in MATLAB (R2010a) on an Intel ${ }^{\circledR}$ Core $^{\mathrm{TM}} 2 \mathrm{CPU}$ machine with a $3.6 \mathrm{GHz}$ processor and $16 \mathrm{~GB}$ of RAM.

\subsection{Initialized parameters}

Table 1 provides the initial parameters of two given Halo orbits around the $L_{1}$ and $L_{2}$ libration points in the Sun-Earth system. The performance analysis and comparison for spacecraft transferred from the Halo orbit around the $L_{1}$ libration point to the Halo orbit around the $L_{2}$ libration point have been given substantial attention, and the case of spacecraft transferred from the $L_{2}$ to $L_{1}$ libration points has been taken as the expanding applications of the proposed methodology. From Table 1, the Jacobi constants of these two Halo orbits are 3.0007481291 and 3.0007380270, and there are no heteroclinic connections. Moreover, the mass ratio is $\mu=3.003477641 \times 10^{-6}$.

Table 2 summarizes the constraint conditions on the design variables. To guarantee a high efficiency by the multi-objective optimization and to obtain more Pareto points, a proper range of each design variable should be determined. The first two design variables ( $t p_{1}$ and $\left.t p_{2}\right)$ are the flight times on Halo orbits, and they determine the spacecraft's starting and ending points. Thus, the flight time of a spacecraft on Halo orbits is one periodic time, i.e., the ranges of these two variables are 0-180 days. The third and fourth design variables $\left(t f_{1}\right.$ and $t f_{2}$ ) are the flight times on the invariant manifolds. If these two design variables are set as 0 , no invariant manifold is used in the spacecraft's transfer trajectories. Thus, the results of multi-objective optimization only contain the transfer trajectories obtained using low-thrust control, and the superiority of the invariant manifold has not been utilized. However, flight times on the invariant manifolds of an excessive number of days may lead to a large distance derivation from the desired ending point, which increases the transfer times and fuel consumption. Therefore, these two 
design variables are in the range of 0-250 days in the following numerical simulations. The last design variable $(t z)$ is the flight time controlled via low-thrust control. The smaller flight times may result in greater fuel consumption; however, the longer flight times are likely to increase the transfer time. Accordingly, the flight time controlled via low-thrust control is taken from 30 days to 100 days to establish a balance between transfer time and fuel consumption.

In addition, the parameters of the multi-objective optimization adaptive surrogate model should be determined. Because a small $F_{\max }$ most likely leads to a higher accuracy solution in exchange for increased computation time, and because more Pareto points may increase the computation time, the parameters are taken as $F_{\max }=1.005$ and $c=40$ to balance the above two aspects.

Table 1 Initial conditions of two Halo orbits around $L_{1}$ and $L_{2}$ libration points

\begin{tabular}{|c|c|c|c|}
\hline Reference Halo orbits & Parameter & Value & Unit \\
\hline \multirow{6}{*}{ around $L_{1}$ libration point } & $x_{0}$ & $1.4795628143 \times 10^{11}$ & $\mathrm{~m}$ \\
\hline & $y_{0}$ & 0 & $\mathrm{~m}$ \\
\hline & $z_{0}$ & $5.9591614488 \times 10^{11}$ & $\mathrm{~m}$ \\
\hline & $\dot{x}_{0}$ & 0 & $\mathrm{~m} / \mathrm{s}$ \\
\hline & $\dot{y}_{0}$ & $3.2371856095 \times 10^{2}$ & $\mathrm{~m} / \mathrm{s}$ \\
\hline & $\dot{z}_{0}$ & 0 & $\mathrm{~m} / \mathrm{s}$ \\
\hline \multirow{6}{*}{ around $L_{2}$ libration point } & $x_{0}$ & $1.5072430333 \times 10^{11}$ & $\mathrm{~m}$ \\
\hline & $y_{0}$ & 0 & $\mathrm{~m}$ \\
\hline & $z_{0}$ & $4.1184521298 \times 10^{11}$ & $\mathrm{~m}$ \\
\hline & $\dot{x}_{0}$ & 0 & $\mathrm{~m} / \mathrm{s}$ \\
\hline & $\dot{y}_{0}$ & $3.765907717 \times 10^{2}$ & $\mathrm{~m} / \mathrm{s}$ \\
\hline & $\dot{z}_{0}$ & 0 & $\mathrm{~m} / \mathrm{s}$ \\
\hline
\end{tabular}

Table 2 Constraint conditions for design variables

\begin{tabular}{cccc}
\hline Design variables & Lower boundary & Upper boundary & Unit \\
\hline$t p_{1}$ & 0 & 180 & day \\
$t p_{2}$ & 0 & 180 & day \\
$t f_{1}$ & 0 & 250 & day \\
$t f_{2}$ & 0 & 250 & day \\
$t z$ & 30 & 100 & day \\
\hline
\end{tabular}


Furthermore, to appraise the optimization results, the 2-norm of the objective functions associated with design points are proposed as the evaluation criterion (Blasco et al., 2008):

$$
\left\|F_{j}\right\|_{2}=\sqrt{\sum_{i=1}^{n} f_{p i}^{j}}
$$

where $f_{p i}^{j}$ is the normalized value for the $i$ th objective function associated with the $j$ th design point.

Under this evaluation criterion and using the hypothesis of equal importance for different objective functions, the smaller $\left\|F_{j}\right\|_{2}$ is for a design point, the better this design point.

\subsection{Results and discussion}

\subsubsection{Performance of adaptive surrogate model based multi-objective optimization}

With the above known information, multi-objective transfer trajectory optimization based on the adaptive surrogate model has been successfully implemented in numerical simulations. The convergence, effectiveness and computational cost of the proposed method should be discussed in detail.

Fig. 5 shows the convergence of the adaptive surrogate model-based multi-objective optimization. It can be seen that most of the initial approximate Pareto points are far from the final approximate Pareto points, and the approximate Pareto points continuously improve during the iteration procedure. In the first few iterations, the Pareto frontier of multi-objective optimization is not clear. Later, a clear Pareto frontier appears in the final several iterations. The iteration procedure is an updating procedure of the mixed sampling strategy, and the added sample points selected by the mixed sampling strategy are combined with the current approximate Pareto points to construct the new surrogate model. In each iteration of the adaptive updating procedure, the good sample points among the current approximate Pareto points have been preserved, and others are discarded. More importantly, all the sample points among the approximate Pareto points have been evaluated using the real multi-objective transfer trajectory optimization model. Thus, all the responses according to these added sample points are real responses of the original multi-objective transfer trajectory optimization model.

Fig. 6 presents a comparison between the pseudo Pareto points and the approximate Pareto points to illustrate the effectiveness of the adaptive surrogate model-based multi-objective optimization. The pseudo Pareto points that are not evaluated through the real multi-objective optimization model are directly obtained from the basic surrogate model without applying adaptive procedures. The approximate 
Pareto points that are evaluated through the real multi-objective optimization model are obtained from the adaptive surrogate model. As seen in Fig. 6, the approximate Pareto points provide a lower transfer time and fuel consumption compared to the pseudo Pareto points, especially for the cases of low transfer times. In addition, the approximate Pareto points present better uniformity and a wider distribution. Therefore, the adaptive surrogate model-based multi-objective optimization proposed in this paper obtains a higher accuracy than does the traditional surrogate model-based method.

Fig. 7 presents the Pareto frontier comparison between the adaptive surrogate model-based multiobjective optimization and the direct multi-objective optimization. In general, the two methods provide results with the same tendencies. In the domain of low transfer time solutions, the adaptive surrogate model-based multi-objective optimization provides better performance. However, in the domain of low fuel consumption solutions, the direct multi-objective optimization provides better performance. The main reason for this is that the sampling guidance function used in this paper is the Pareto fitness function and the maximum crowding distance. Therefore, the domain of low fuel consumption solutions lies in a small range of the Pareto frontier. In addition, this range lies on the edge of the front, and the Pareto fitness function does not obtain the largest value. The points for the maximum crowding distance do not have a substantial effect with increasing number of Pareto points.

Figs. 8 and 9 present the 2-norm level diagrams for the two objective functions, i.e., the total transfer time and the total fuel consumption. The 2-norms of the total transfer time and the total fuel consumption are evaluated using the distance between the normalized objective function and minimum normalized objective function. In Figs. 8 and 9, the blue stars represent the approximate Pareto points, and the red circles represent all the sample points. Because most of the approximate Pareto points are at the periphery of level diagrams, the high efficiency of the mixed sampling strategy is proved. Moreover, there are obvious blank regions, or discontinuities, between 0.2 and 0.5 on the 2 -norms of the total transfer time and the total fuel consumption. These blank regions, or discontinuities, from Figs. 8 and 9 correspond to similar phenomena in Figs. 5 and 6 with the correct time and space position. Therefore, when the design variables can be chosen, the values of the objective functions cannot be obtained in the above regions. 


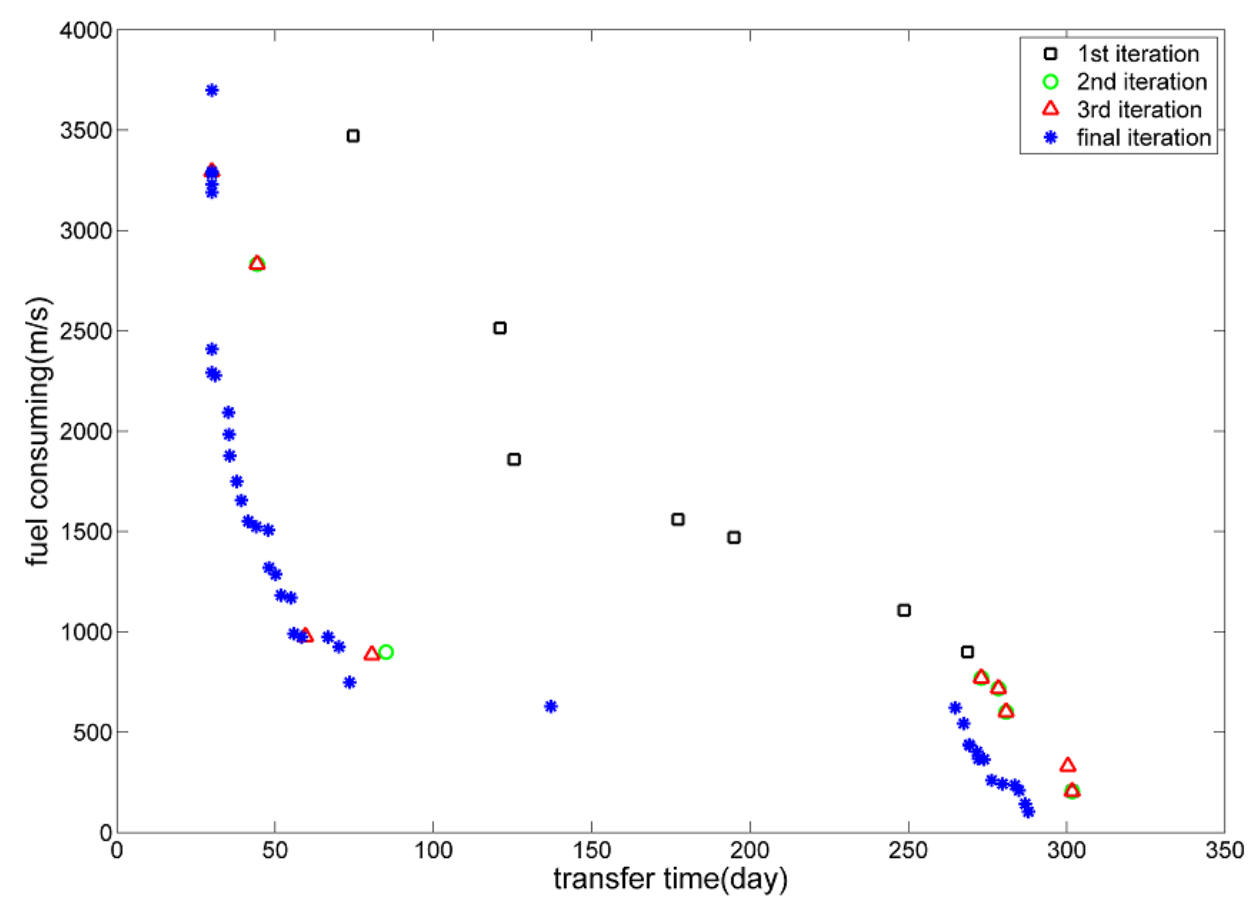

Fig.5. Convergence of the adaptive surrogate model based multi-objective optimization

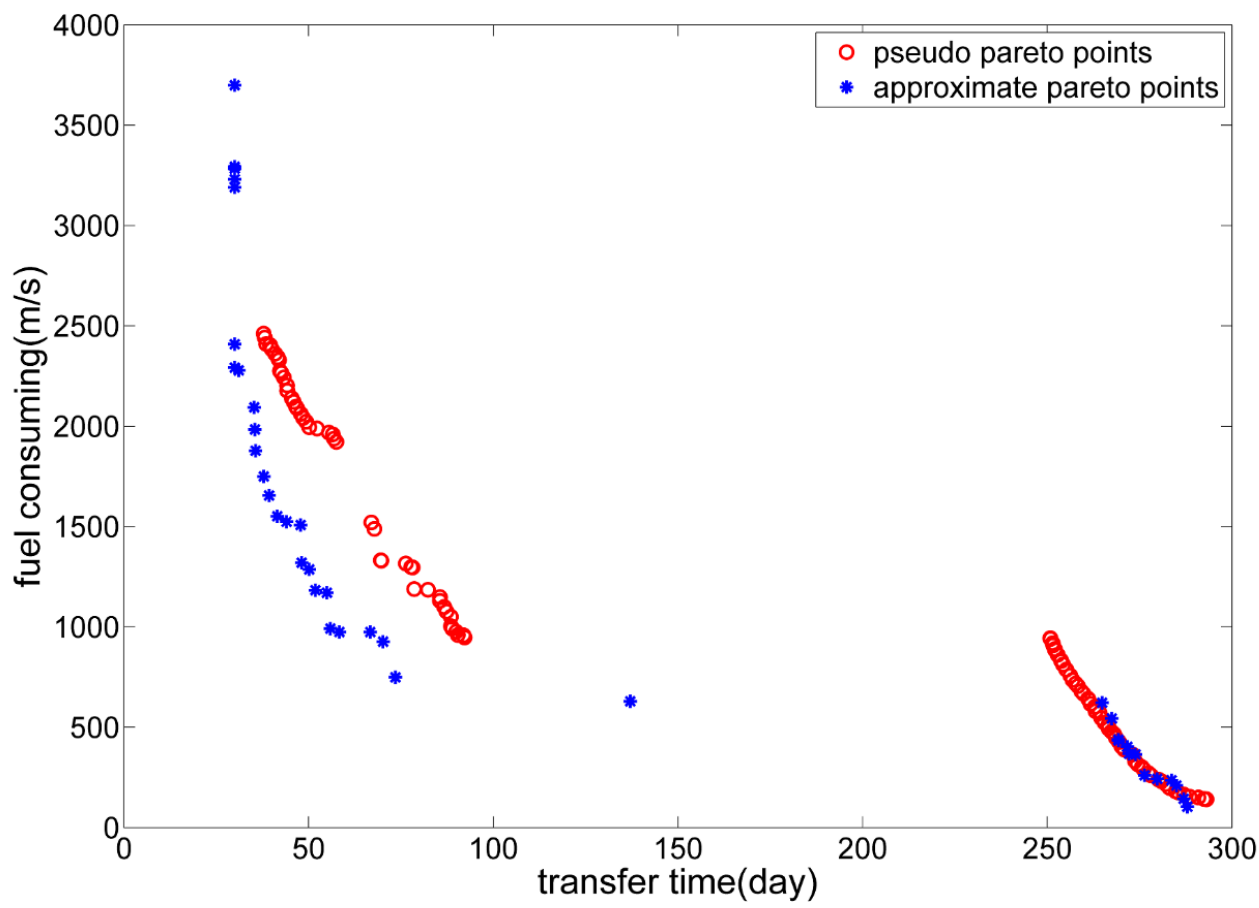

Fig.6. Comparison between the pseudo Pareto points and the approximate Pareto points 


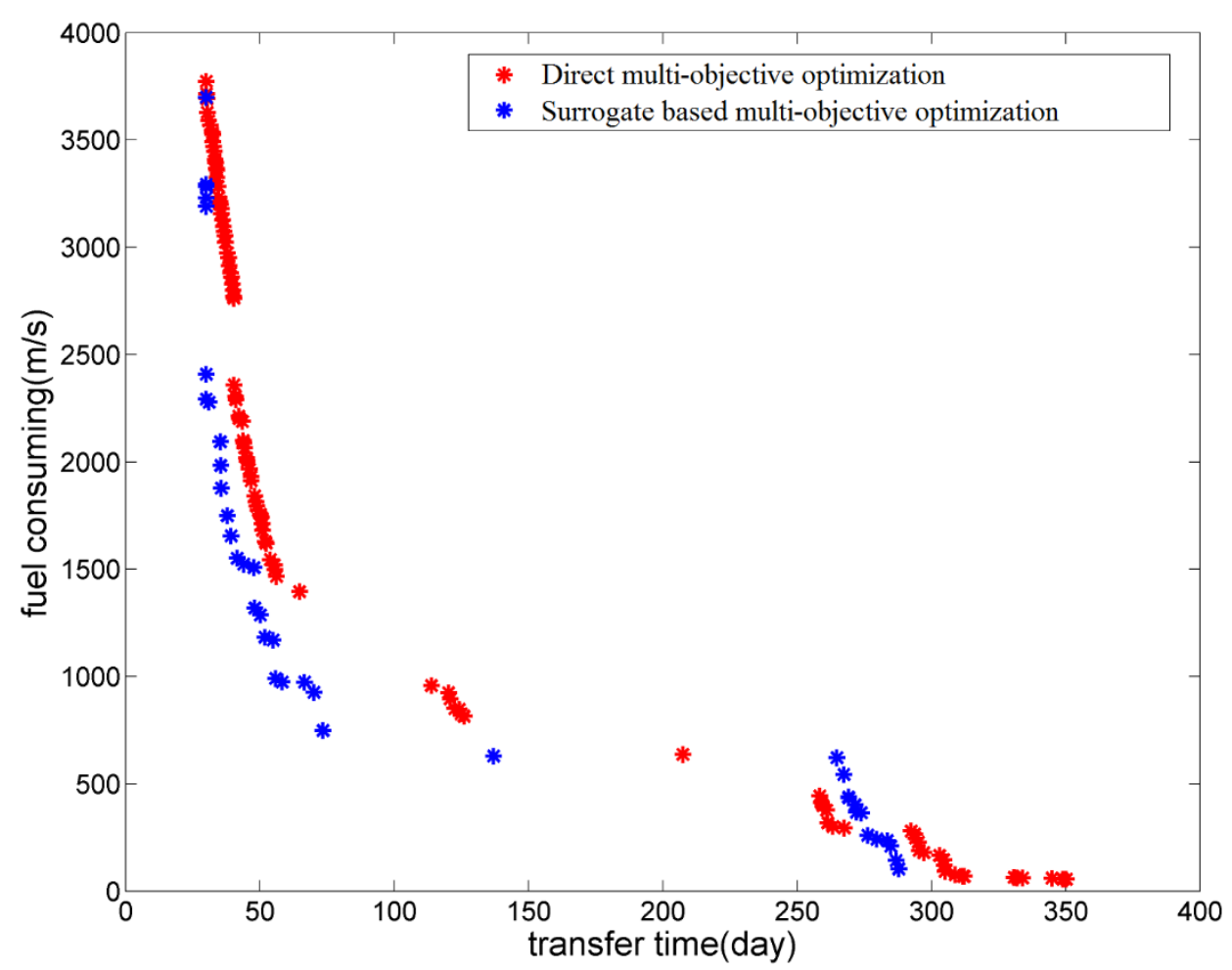

Fig.7. Pareto frontier comparison between the adaptive surrogate model based multi-object optimization and direct multi-object optimization

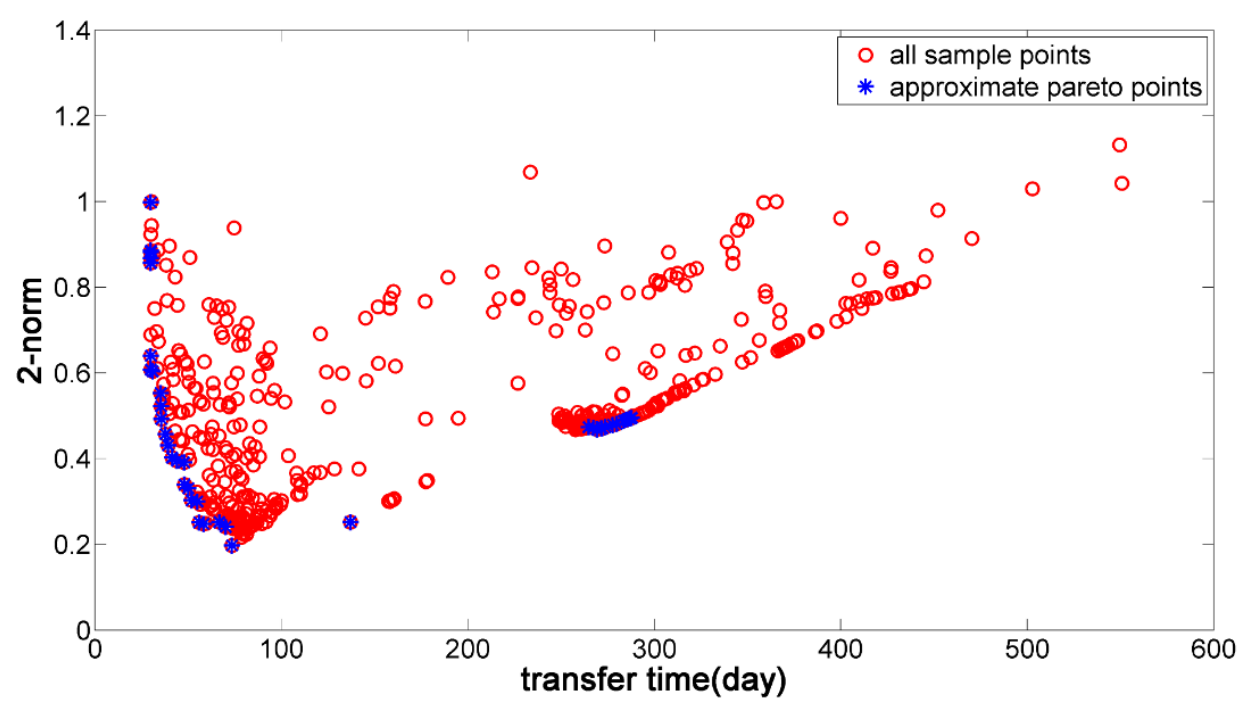

Fig.8. 2-norm level diagram representation of transfer time 


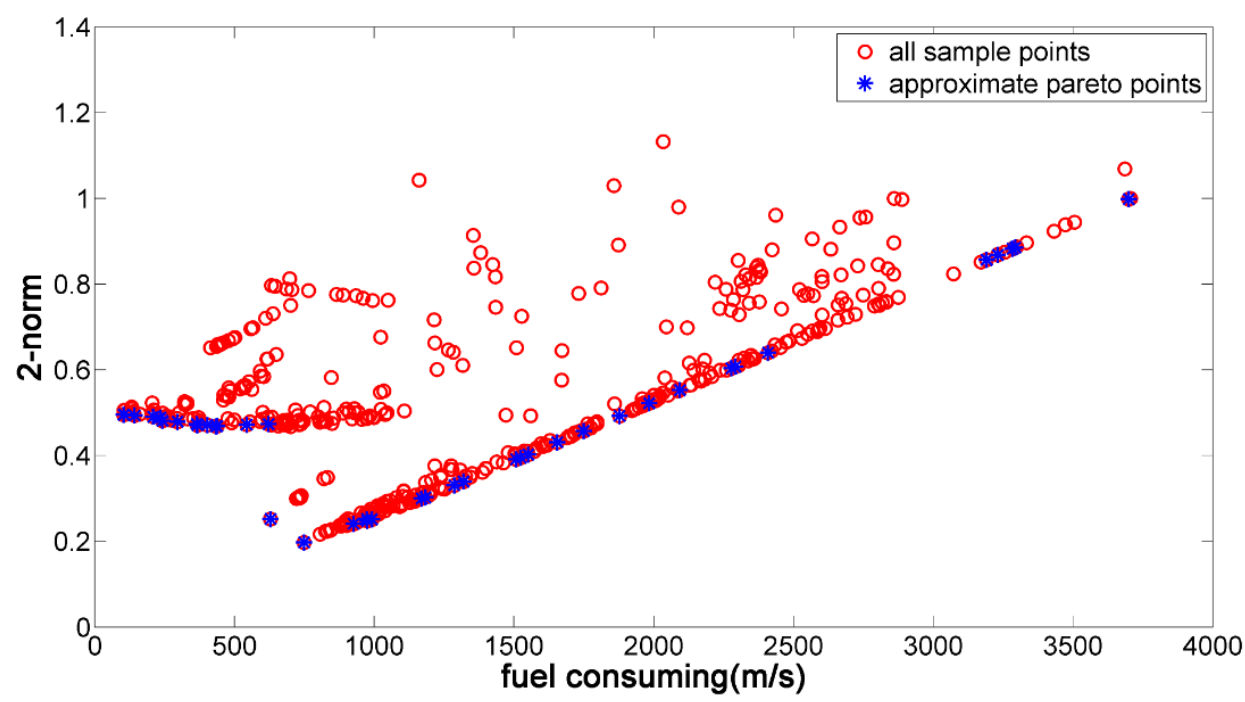

Fig.9. 2-norm level diagram representation of fuel consumption

Table 3 compares the results of both the adaptive surrogate model and the direct optimization model. The proposed adaptive surrogate model-based multi-objective optimization method has obtained 41 approximate Pareto points at the cost of evaluating 479 sample points. Direct multi-objective optimization without the surrogate model has obtained 100 approximate Pareto points at the cost of evaluating 10000 sample points. The efficiency of generating Pareto points using the proposed method is 8.56 times that of the direct multi-objective optimization method. More importantly, the computational cost from the perspective of evaluating sample points has been substantially decreased by the proposed adaptive surrogate model; a computational cost of $4.79 \%(479 / 10000=4.79 \%)$ of that of the direct multiobjective optimization method is obtained. Based on the detailed CPU time results obtained using these two methods, we can see that the CPU time for the adaptive surrogate model-based multi-objective optimization is 2764 s. This time includes two parts, i.e., the CPU time for generating the adaptive surrogate model (1293 s) and the CPU time for implementing the optimization solver with the adaptive surrogate model (1471 s). Therefore, the total computational time of the adaptive surrogate model-based multi-objective optimization is approximately $10.10 \%(2764 / 27362 \approx 10.10 \%)$ of that of the direct multiobjective optimization method.

Table 3 Results comparison between the adaptive surrogate model and the original model

\begin{tabular}{lcccc}
\hline Methods & $\begin{array}{c}\text { Number of } \\
\text { evaluated points }\end{array}$ & $\begin{array}{c}\text { Number of } \\
\text { Pareto points }\end{array}$ & $\begin{array}{c}\text { Efficiency of } \\
\text { generating Pareto }\end{array}$ & CPU time(s) \\
\hline $\begin{array}{l}\text { Adaptive surrogate } \\
\text { model optimization } \\
\text { Direct optimization }\end{array}$ & 479 & 41 & $41 / 479 \approx 8.56 \%$ & $1293+1471=2764$ \\
\hline
\end{tabular}




\subsubsection{Comparison of multi-objective transfer trajectories}

The convergence, effectiveness and computational cost of the proposed adaptive surrogate model have been studied through the above numerical simulations. The multi-objective optimization results of spacecraft transferring from a Halo orbit around the $L_{1}$ libration point to a Halo orbit around the $L_{2}$ libration point should also be explained in detail. Fig. 10 shows many different transfer trajectories for different approximate Pareto points. Table 4 lists the detailed results of the design variables and objective functions for cases $1-5$.

From cases 1, 2 and 3, according to Fig. 10 and Table 4, the transfer trajectories of a spacecraft are mainly dominated by low-thrust control, and only a few small invariant manifolds have been employed in these transfer trajectories. Meanwhile, the approximate Pareto points distributed in the range of 0-70 transfer days also have the same feature. This means that the transfer trajectories of the spacecraft utilize almost no invariant manifolds. Furthermore, when the transfer time is in the range of 70-260 days, almost no approximate Pareto points exist with increased transfer time. Although invariant manifolds have been employed in transfer trajectories in the blank region of the approximate Pareto frontier, the fuel consumption has not obviously decreased. The main reason for this is that the lengths of invariant manifolds remain insufficient, and the distances between the invariant manifolds and Halo orbits remain small. Another important phenomenon can be observed in cases 4 and 5. The invariant manifolds of Halo orbits around the $L_{1}$ libration point have not been fully utilized, and only the invariant manifolds of Halo orbits around the $L_{2}$ libration point have been fully utilized. There are two main reasons for this phenomenon. The first reason is that the invariant manifolds of Halo orbits around the $L_{1}$ libration point do not have a substantial influence when the transfer time is less than one period of the Halo orbit. To fully utilize both invariant manifolds associated with Halo orbits around the $L_{1}$ and $L_{2}$ libration points, the transfer time needs to be increased significantly. The other reason is that one invariant manifold of a Halo orbit around the $L_{2}$ libration point can already guarantee a short trajectory under low-thrust control, and there is no need for the other invariant manifolds. Therefore, invariant manifolds are fully utilized in cases 4 and 5, and the fuel consumption obviously decreases. Cases 4 and 5 show the fuel consumption benefit of employing invariant manifolds in the transfer trajectories.

To further demonstrate the benefits of invariant manifolds, two types of transfer technologies, i.e., 
invariant manifolds combined with low-thrust control and pure low-thrust control, have been compared. Under the same initial parameters and total transfer time, the fuel consumption of spacecraft with/without using invariant manifolds has been summarized in Table 5. From Table 5, a spacecraft with invariant manifolds combined with low-thrust control saves substantial fuel compared with only using low-thrust control. Although the total transfer time is the same for the above two types of transfer technologies, the flight time controlled under low-thrust control in the mixed transfer method is substantially smaller than that under pure low-thrust control. Therefore, the fuel consumption of a spacecraft transfer mission is not a monotonic function of the controlled flight time. The goal of reducing fuel consumption cannot always be achieved with increasing controlled flight time, and larger controlled flight times most likely lead to longer transfer trajectories.

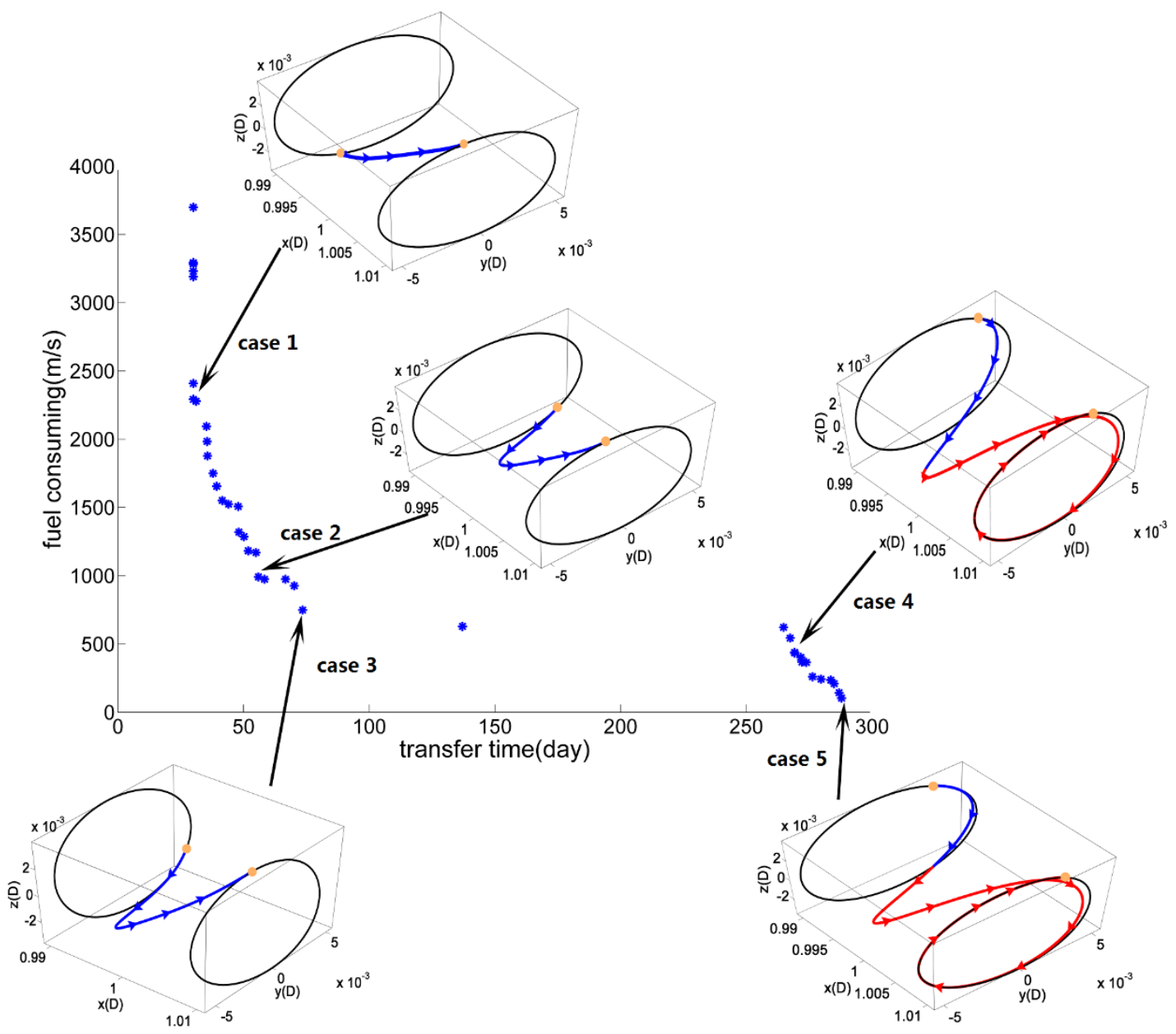

Fig.10. Transfer trajectories for different approximate Pareto points

Table 4 Detailed results of design variables and objective functions for cases 1-5

\begin{tabular}{|c|c|c|c|c|c|}
\hline Parameters & Case 1 & Case 2 & Case 3 & Case 4 & Case 5 \\
\hline
\end{tabular}




\begin{tabular}{clllll}
\hline$t p_{1}($ day $)$ & 97.0749 & 71.5077 & 66.1213 & 24.4944 & 21.0556 \\
$t p_{2}($ day $)$ & 179.9962 & 180.0000 & 180.0000 & 17.2648 & 21.3464 \\
$t f_{1}($ day $)$ & 0.0000 & 0.0000 & 0.1908 & 0.0000 & 0.0000 \\
$t f_{2}($ day $)$ & 0.0000 & 0.0000 & 0.0240 & 221.6268 & 238.2576 \\
$t z($ day $)$ & 31.0807 & 55.8968 & 73.2942 & 47.5851 & 49.6031 \\
$\Delta t($ day $)$ & 31.0807 & 55.8968 & 73.5090 & 269.2119 & 287.8608 \\
$\Delta v(\mathrm{~m} / \mathrm{s})$ & 2278.0495 & 991.0967 & 748.6899 & 438.0504 & 104.1134 \\
\hline
\end{tabular}

Table 5 Comparisons between mixed transfer manner and pure low-thrust

\begin{tabular}{lcccc}
\hline \multirow{2}{*}{ Parameters } & \multicolumn{3}{c}{ Case 4 } & \multicolumn{2}{c}{ Case 5 } \\
& Mixed transfer & Pure low-thrust & Mixed transfer & Pure low-thrust \\
\hline$t p_{1}$ (day) & 24.4944 & 24.4944 & 21.0556 & 21.0556 \\
$t p_{2}$ (day) & 17.2648 & 17.2648 & 21.3464 & 21.3464 \\
$t f_{1}($ day $)$ & 0 & 0 & 0 & 0 \\
$t f_{2}($ day $)$ & 221.6268 & 0 & 238.2576 & 0 \\
$t z$ (day) & 47.5851 & $47.5851+221.6268$ & 49.6031 & $49.6031+238.2576$ \\
$\Delta t($ day $)$ & 269.2119 & 269.2119 & 287.8608 & 287.8608 \\
$\Delta v(\mathrm{~m} / \mathrm{s})$ & 438.0504 & 1646.4007 & 104.1134 & 1628.3900 \\
\hline
\end{tabular}

\subsubsection{Expanding applications}

The performance of the adaptive surrogate model and the comparison of the results of the multiobjective transfer trajectories have been discussed in the previous subsections. The expanding application of the proposed method can be implemented in this subsection, and the robustness of the proposed method can also be tested using the expanding application. For convenience, the trip of a spacecraft transferred from a Halo orbit around the $L_{1}$ libration to a Halo orbit around the $L_{2}$ libration is called the going trip. The opposite trip is conversely called the returning trip. With the initial Halo orbit of the going trip taken as the target Halo orbit of the returning trip, 49 approximate Pareto points of the returning trip have been obtained using the presented adaptive surrogate model-based multi-objective transfer trajectory optimization at the cost of evaluating 443 sample points. Fig. 11 shows the approximate Pareto points and some typical transfer trajectories of both the going trip and the returning trip. 


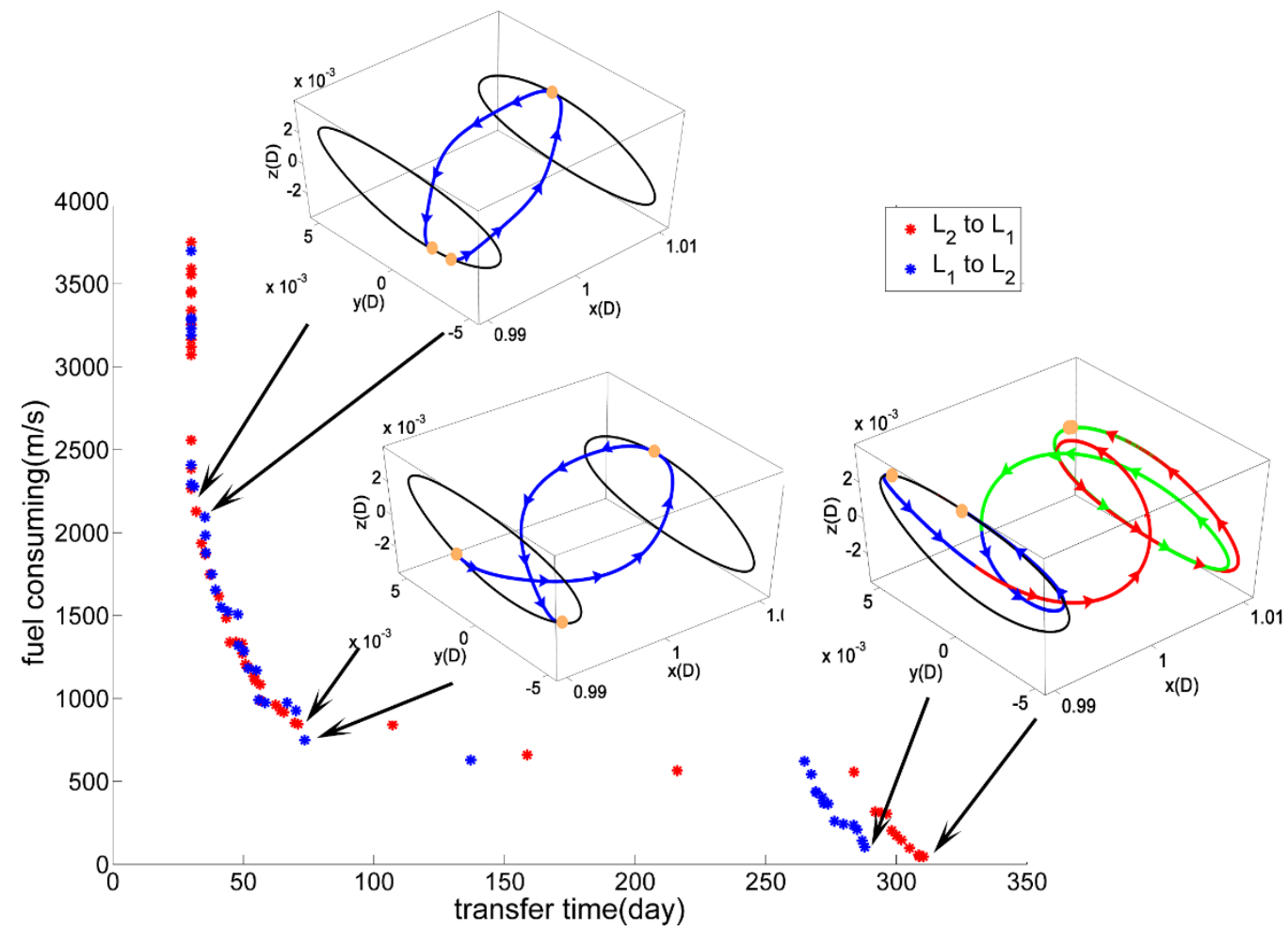

Fig. 11. Comparison of the going trip and the returning trip

Fig. 11 shows that almost identical approximate Pareto points are obtained for both the going trip and the returning trip with small total transfer times, and a slight difference arises between the going trip and the returning trip with large total transfer times. Moreover, an interesting phenomenon is observed whereby three typical pairs of going trips and returning trips obtain almost symmetric transfer trajectories, especially for small total transfer times. In the case of small total transfer times, because the invariant manifolds have not been fully utilized and because the main transfer time has been dominated by the low-thrust control, the approximate Pareto points are almost the same for the above two cases. In the case of large total transfer times, the invariant manifolds and low-thrust control have been fully utilized, a minimal difference can be observed for the above two cases. In particular, under the same total transfer time, the going trip uses less fuel than does the returning trip. With a slightly longer total transfer time, the fuel consumption of the returning trip can be further reduced compared to that of the going trip. It is noted that the potential energy around the $L_{1}$ libration point and around the $L_{2}$ libration point exhibit a slight difference; in addition, the going trip is directed toward the Earth, and the returning trip is directed far away from the Earth. These slight disturbances may have a slight influence under long transfer times. The reasons for this small difference should be further studied in the future. 


\section{Conclusions}

An adaptive surrogate model-based multi-objective optimization for mixed spacecraft transfer trajectory design is proposed for effectively obtaining the approximate Pareto points of spacecraft transferring between Halo orbits around the $L_{1}$ and $L_{2}$ libration points in the Sun-Earth system. The efficiency of the proposed method in obtaining approximate Pareto points is 8.56 times that of the direct multi-objective optimization method while requiring only $10.10 \%$ of the computational time of that method. Compared with the basic surrogate model, the approximate Pareto points of the proposed method have been verified through the real multi-objective optimization model, and the optimality of the present adaptive surrogate model can be guaranteed. Therefore, the convergence, effectiveness and computational cost of the proposed adaptive surrogate model provide obvious advantages. Furthermore, with the same approximate Pareto points, spacecraft transfer problems with mixed transfer technologies, i.e., invariant manifolds combined with low-thrust control and pure low-thrust control, have been compared. There is an obvious fuel consumption benefit of employing invariant manifolds in the design of multi-objective transfer trajectories. Finally, the robustness of the proposed method has also been tested by the expanding application.

\section{Acknowledgements}

The authors are grateful for the financial support of the National Science Foundation of China (11472069, 11432010); the Project Funded by China Postdoctoral Science Foundation (2014M550155, 2015T80245); and the Dalian Science and Technology Project (2015A11GX037).

\section{References}

Assadian, N., Pourtakdoust, S.H. Multiobjective genetic optimization of Earth-Moon trajectories in the restricted four-body problem, Adv. Space Res. 45(3), 398-409, 2010.

Blasco, X., Herrero, J.M., Sanchis, J., et al. A new graphical visualization of n-dimensional Pareto front for decision-making in multiobjective optimization. Inf. Sci. 178(20), 3908-3924, 2008.

Coffee, T.M., Anderson, R.L., Lo, M.W. Multiobjective optimization of low-energy trajectories using 
optimal control on dynamical channels, AAS/AIAA Space Flight Mechanics Meeting, AAS 11-129, 2011.

Coverstone-Carroll, V., Hartmann, J.W., Mason, W.J. Optimal multi-objective low-thrust spacecraft trajectories, Comput. Meth. Appl. Mech. Eng. 186(2-4), 387-402, 2000.

Cai, Z., Li, X., Wu, Z. Deployment and retrieval of a rotating triangular tethered satellite formation near libration points, Acta. Astronaut. 98, 37-49, 2014.

Davis, K.E., Anderson, R.L., Scheeres, D.J., et al. The use of invariant manifolds for transfers between unstable periodic orbits of different energies, Celest. Mech. Dyn. Astron. 107(4), 471-485, 2010.

Davis, K.E., Anderson, R.L., Scheeres, D.J., et al. Optimal transfers between unstable periodic orbits using invariant manifolds, Celest. Mech. Dyn. Astron. 109(3), 241-264, 2011.

Dankanich, J.W. Low-Thrust Mission Design and Application, in: Proceedings of the $46^{\text {th }}$ AIAA/ASME/SAE/ASEE Joint Propulsion Conference \& Exhibit, TN, AIAA 2010-6857, 2010.

Dellnitz, M., Ober-Blöbaum, S., Post, M., et al. A multi-objective approach to the design of low thrust space trajectories using optimal control, Celest. Mech. Dyn. Astron. 105(1-3), 33-59, 2009.

Deb, K., Pratap, A., Agarwal, S., et al. A fast and elitist multi objective genetic algorithm NSGA-II. IEEE Trans. Evol. Comput. 6(2), 182-197, 2002.

Forrester, A.I J., Keane, A.J. Recent advances in surrogate-based optimization, Prog. Aeosp. Sci. 45(13), 50-79, 2009.

Gano, S.E., Kim, H., Brown II, D.E. Comparison of three surrogate modeling techniques: datascape, kriging, and second order regression, 11th AIAA/ISSMO Multidisciplinary Analysis and Optimization Conference, AIAA 2006-7048, 2006.

Gómez, G., Jorba, A., Masdemont, J., et al. Study of the transfer from the earth to a halo orbit around the equilibrium point L1. Celest. Mech. Dyn. Astron. 56(4), 541-562, 1993.

Gómez, G., Koon, W.S., Marsden, J.E., et al. Connecting orbits and invariant manifolds in the spatial restricted three-body problem, Nonlinearity 17(5), 1571-1606, 2004.

Howell, K.C., Kakoi, M. Transfers between the Earth-Moon and Sun-Earth systems using manifolds and transit orbits, Acta. Astronaut. 59(1-5), 367-380, 2006.

Peng, H.J., Jiang, X., Chen, B. S. Optimal nonlinear feedback control of spacecraft rendezvous with finite low-thrust between libration orbits, Nonlinear Dyn. 76(2), 1611-1632, 2014.

Peng, H.J., Zhao, J., Wu, Z.G., et al. Optimal periodic controller for formation flying on libration point 
orbits, Acta. Astronaut. 69 (7-8), 537-550, $2011 \mathrm{a}$.

Peng, H.J., Gao, Q., Wu, Z.G., et al. Optimal guidance based on receding horizon control for low-thrust transfer to libration point orbits, Adv. Space Res. 51(11), 2093-2111, 2013.

Pehlivanoglu, Y.V., Yagiz, B. Aerodynamic design prediction using surrogate-based modeling in genetic algorithm architecture, Aerosp. Sci. Technol. 23(1), 479-491, 2012.

Peng, H.J., Yang, C.F., Li, Y.P., et al. Surrogate-based parameter optimization and optimal control for optimal trajectory of Halo orbit rendezvous, Aerosp. Sci. Technol. 26(9), 176-184, 2013.

Peng, H.J., Gao, Q., Wu, Z.G., et al. Symplectic adaptive algorithm for solving nonlinear two-point boundary value problems in astrodynamics, Celest. Mech. Dyn. Astron. 110(4), 319-342, 2011 b.

Qi, R., Xu, S. Optimal low-thrust transfers to lunar L1 halo orbit using variable specific impulse engine, J. Aerosp. Eng. 28(4), 04014096, 2015.

Queipo, N.V., Haftka, R.T., Shyy, W., et al. Surrogate-based analysis and optimization, Prog. Aeosp. Sci. 41(1), 1-28, 2005.

Rayman, M.D., Varghese, P., Lehman, D.H., et al. Results from the deep space 1 technology validation mission, Acta. Astronaut. 47 (2-9), 475-487, 2000.

Schaumann, E.J., Balling, R.J., Day, K. Genetic algorithms with multiple objectives, 7th AIAA Symposium on Multidisciplinary Analysis and Optimization, AIAA-98-4974, 1998.

Shan, S., Wang, G.G. An efficient pareto set identification approach for multiobjective optimization on multi-object transfer models, J. Mech. Des. 127(5), 866-874, 2005.

Serban, R., Koon, W.S., Lo, M.W., et al. Halo orbit mission correction maneuvers using optimal control. Automatica. 38(4), 571-583, 2002.

Schütze, O., Vasile, M., Junge, O., et al. Designing optimal low-thrust gravity-assist trajectories using space pruning and a multi-objective approach, Eng. Optimiz. 41(2). 155-181, 2009.

Szebehely, V. Theory of Orbits. Academic Press, New York, 1967.

Vaquero, M., Howell, K.C. Leveraging resonant-orbit manifolds to design transfers between librationpoint orbits, J. Guid. Control Dyn. 37(4), 1143-1157, 2014.

Zhao, J., Cai, Z. Nonlinear dynamics and simulation of multi-tethered satellite formations in Halo orbits, Acta. Astronaut. 63(5-6), 673-681, 2008. 